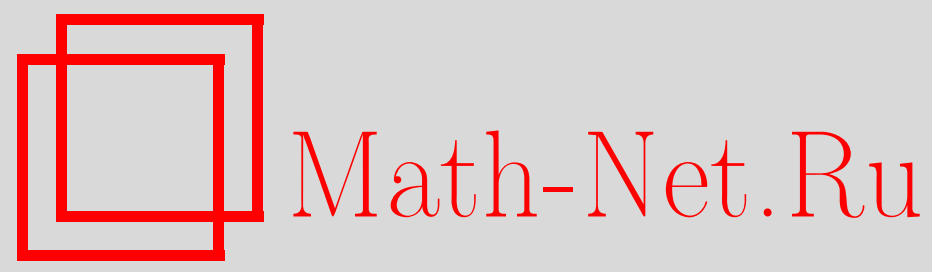

А. Б. Богатырев, Многообразия опорных множеств многочленов Чебышева, Матем. заметки, 2000, том 67, выпуск $6,828-836$

DOI: https://doi.org/10.4213/mzm901

Использование Общероссийского математического портала Math-Net.Ru подразумевает, что вы прочитали и согласны с пользовательским соглашением http://www . mathnet.ru/rus/agreement

Параметры загрузки:

IP : 52.90 .164 .192

26 апреля 2023 г., 12:21:22

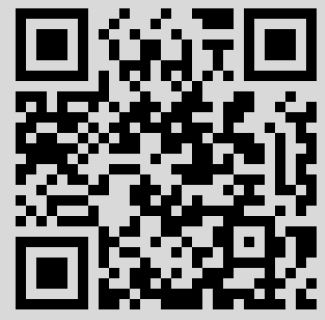




\section{МНОГООБРАЗИЯ ОПОРНЫХ МНОЖЕСТВ МНОГОЧЛЕНОВ ЧЕБЫШЕВА}

\section{А. Б. Богатырев}

В работе рассмотрена параметризация совокупности многочленов $T_{n}(E, x)$, наименее уклоняющихся от нуля на системе $E$ из нескольких отрезков действительной оси. Дан новый вывод уравнений, определяющих границу максимального множества наименьшего уклонения $E^{+} \supset E$. Описана геометрия многообразия всевозможных множеств $E^{+}$, вложенного в пространство модулей гиперэллиптических кривьх с действительными точками ветвления.

Библиография: 9 названий.

1. Введение. Пусть $E$ - содержащее более $n$ точек множество, полученное выбрасьванием нескольких интервалов из отрезка $[-1,1]$. Многочленом Чебышева множества $E$ назовем многочлен $T_{n}$ степени $n$ с вещественными коэффициентами, имеющий наибольший старший коэффициент среди всех многочленов $P_{n}$, удовлетворяющих условию $\max _{x \in E}\left|P_{n}(x)\right| \leqslant 1$. Теорема Чебышева об альтернансе дает качественное описание графика $T_{n}$ (см. рис. 1 ).

Теорема 1 [1], [2]. Многочлен $P_{n}(x)$ степени п с положительным стариим коәффиииентом является многочленом Чебишева некоторого мнохества Е тогда и только тогда, когда интервал $I:=(-1,1)$ содержит $n$ непересекающ,хся подинтервалов $I_{1}, \ldots, I_{n}$, из которых два примыкают к концам $I$, и таких, что $P_{n}: I_{s} \rightarrow I-$ гомеоморфизм для всех $s=1, \ldots, n$.

Из приведенной теоремы следует, что фиксированньй многочлен Чебышева задает целое семейство множеств $E$, для которых именно он является наименее отклоняющимся от нуля. Следуя [1], мы рассмотрим объекты, находящиеся во взаимно однозначном соответствии с множеством всевозможных многочленов Чебьшева: опорные множества и упорядоченные разбиения степени на натуральные слагаемые. Onорным множеством $E^{+}$многочлена Чебьшева $T_{n}$ назовем множество

$$
E^{+}:=\bigcup_{s=1}^{n} \bar{I}_{s}=T_{n}^{-1}(\bar{I}),
$$

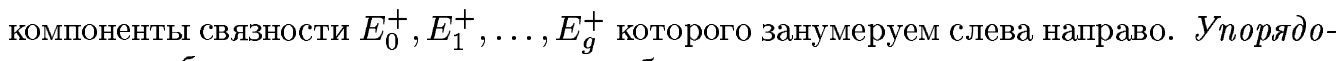
ченное разбиение степени многочлена $n$ образуют числа $m_{k}$, равные количеству подьнтервалов $I_{s}$ в компоненте $E_{k}^{+}$:

$$
m_{0}+m_{1}+\cdots+m_{g}=n .
$$

Работа выполнена при финансовой поддержке Российского фонда фундаментальных исследований, грант № 99-01-00141. 


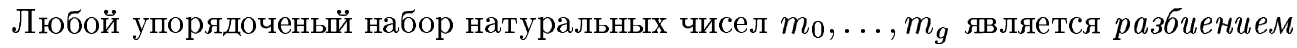
степени для некоторого многочлена Чебьшева. В противоположность этому множество $E^{+}$не произвольно: на его $2 g$ свободных конщов имеется $g$ ограничений, к формулировке которых мы приступаем.

Всякому множеству

$$
\begin{gathered}
E^{+}=:[-1,1] \backslash \bigcup_{k=1}^{g}\left(a_{k}, b_{k}\right), \\
b_{0}:=1<a_{1}<b_{1}<\cdots<a_{g}<b_{g}<1=: a_{g+1},
\end{gathered}
$$

сопоставим (двулистную) риманову поверхность $M=M\left(E^{+}\right)$рода $g$ :

$$
w^{2}=\left(x^{2}-1\right) \prod_{k=1}^{g}\left(x-a_{k}\right)\left(x-b_{k}\right), \quad t=(x, w) \in M\left(E^{+}\right),
$$

на которой введем два канонических базиса гомологий: $\mathbf{A}_{1}, \ldots, \mathbf{A}_{g} ; \mathbf{B}_{1}, \ldots, \mathbf{B}_{g}$ и $\widetilde{\mathbf{B}}_{1}$, $\ldots, \widetilde{\mathbf{B}}_{g} ; \widetilde{\mathbf{A}}_{1}, \ldots, \widetilde{\mathbf{A}}_{g}$. Представители $\mathbf{A}_{s}, \widetilde{\mathbf{B}}_{s}$ показаны на рис. 2, остальные циклы определим равенствами $\mathbf{B}_{s}:=\widetilde{\mathbf{B}}_{0}+\widetilde{\mathbf{B}}_{1}+\cdots+\widetilde{\mathbf{B}}_{s-1} ; \widetilde{\mathbf{A}}_{s}:=\mathbf{A}_{1}+\cdots+\mathbf{A}_{s}$.
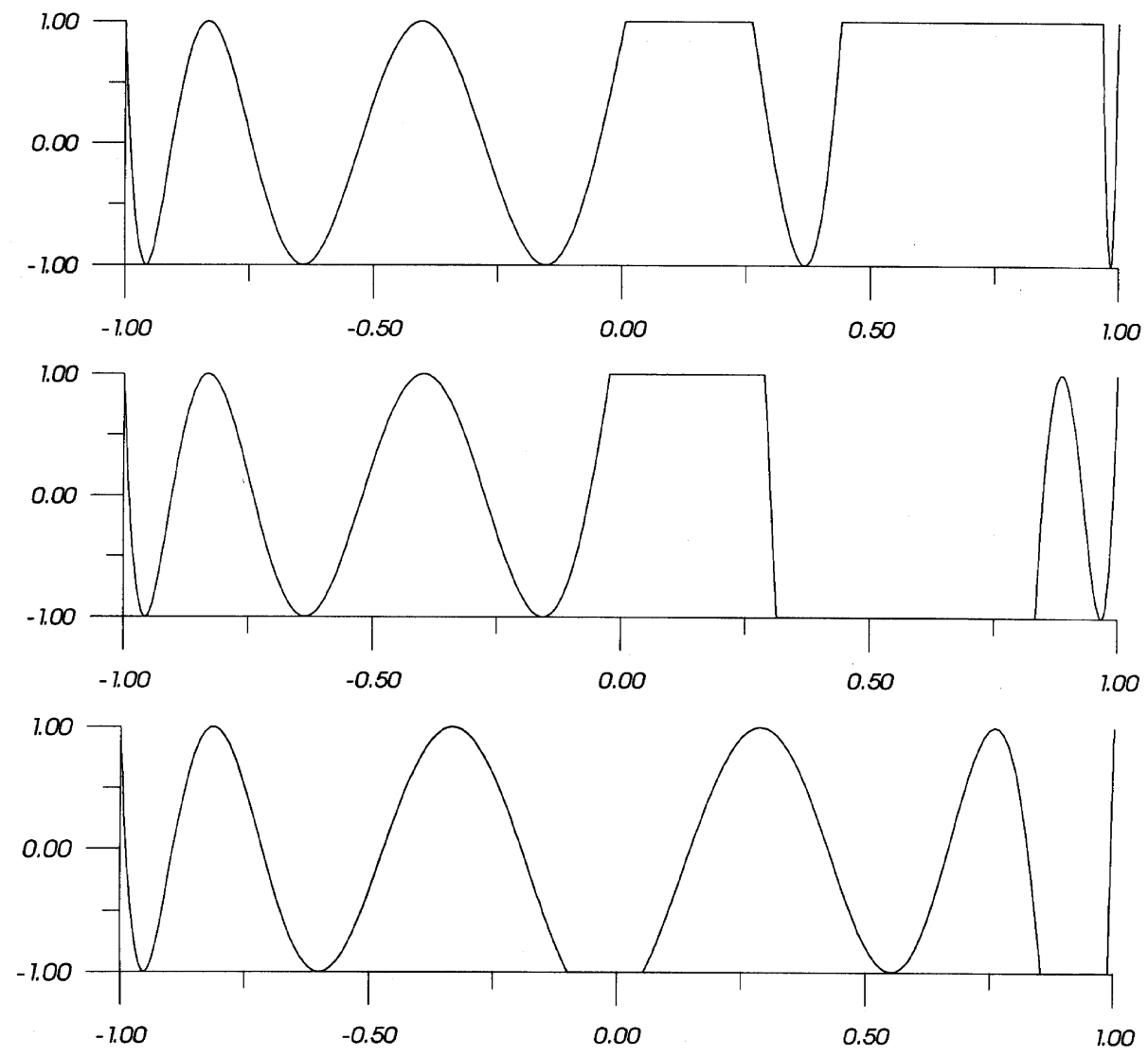

Рис. 1. Многочлены Чебьшева для $g=2, n=10$ и разбиений $6+2+2 ; 6+1+3 ; 5+4+1$ 
Теорема 2 (Уравнения Абеля; [1]-[3]). Множество $E^{+}$является опорным для многочлена Чебышева с разбиением степени (2) тогда и только тогда, когда А-нормированный абелев дифференииал третьего рода $d \eta$ (определение см. в [4]) на римановой поверхности $M\left(E^{+}\right)$с полюсами в двух бесконечно удаленных точках: $\infty_{+}$верхнего листа, $\infty_{-}$нижнего листа и вычетами $\left.\operatorname{Res} d \eta\right|_{ \pm}=\mp 1$, имеет следующие $\widetilde{\mathbf{B}}-$ периоды:

$$
\int_{\widetilde{\mathbf{B}}_{k}} d \eta=2 \pi i \frac{m_{k}}{n}, \quad k=0,1, \ldots, g .
$$

Многочлен Чебышева однозначно восстанавливается по удовлетворяющему (6) опорному мнохеству:

$$
T_{n}\left(E^{+}, x\right)=\cos \left(i n \int_{t_{0}}^{t(x)} d \eta\right), \quad t(x)=(x, w) \in M, \quad t_{0}=(1,0) \in M,
$$

где выражсние для $T_{n}$ не зависит от двузначного выбора $t(x)$ и пути интегрирования на $M$.

ЗАмЕчаниЕ 1. Среди соотношений (6) независимыми являются лишш (любые) $g$, так как контур $\widetilde{\mathbf{B}}_{0}+\widetilde{\mathbf{B}}_{1}+\cdots+\widetilde{\mathbf{B}}_{g}$ стягивается к контуру, огибающему полюс $d \eta$.

ЗАмЕчАниЕ 2. Билинейные соотношения Римана позволяют переписать уравнения (6) в виде

$$
\sum_{k=1}^{g} m_{k} \int_{\tilde{\mathbf{A}}_{k}} d \zeta+n \int_{\infty_{-}}^{\infty_{+}} d \zeta=0
$$

где $d \zeta$ - произвольньй дифференциал первого рода на $M\left(E^{+}\right)$, а интегрирование от $\infty_{-}$ к $\infty_{+}$производится вдоль пути $\mathbf{C}_{0}$ рис. 2 .

ЗАмЕчАниЕ 3. Уравнения (8) в форме соотношений для функций Шоттки при $g=2$ и $m_{1}=m_{2}=1$ применялись Ахиезером в [5] для нахождения многочленов наименьшего отклонения. Формула (7) - интерпретация известного представления многочленов наименьшего отклонения через конформные отображения на гребенчатые области [2], [6]. В используемом здесь виде уравнения (6), (7) введены Пеершторфером в [1].

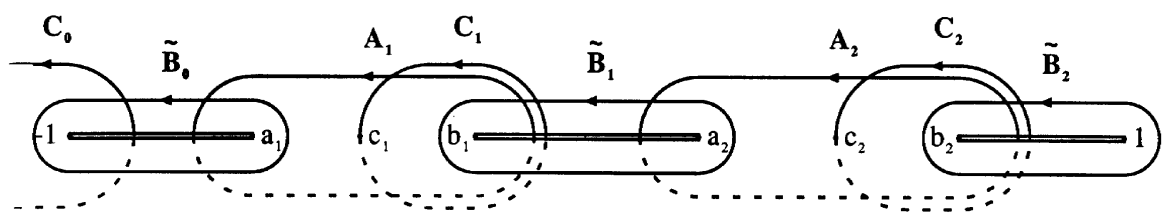

Рис. 2. Циклы $\mathbf{A}_{s}, \widetilde{\mathbf{B}}_{s}$ и пути $\mathbf{C}_{s}$ на римановой поверхности $M\left(E^{+}\right), g=2$

Для геометрического описания совокупности всевозможньх опорных множеств введем два определения.

1) Пространством модулей $\mathscr{H}(\mathbb{R} ; g, 1)$ назовем $2 g$-мерньй симплекс переменных $a_{k}, b_{k}(4)$, точки которого отождествим с римановьми поверхностями $(5)$ с отмеченной точкой $\infty_{+}$.

2) Множество решений уравнений $(6)$ в пространстве $\mathscr{H}(\mathbb{R} ; g, 1)$ обозначим $\mathbb{T}\left(m_{0}, \ldots\right.$, $\left.m_{g}\right)$.

В последнем пункте настояшей заметки доказана 
Теорема 3. Множество $\mathbb{T}\left(m_{0}, \ldots, m_{g}\right)$ - гладкое аналитическое $g$-мерное подмногообразие $\mathscr{H}(\mathbb{R} ; g, 1)$, гомеоморфное клетке. Два многообразия $\mathbb{T}$ либо не пересекаются, либо совпадают; последнее происходит в случае пропорциональности наборов чисел $\left(m_{0}, m_{1}, \ldots, m_{g}\right)$. $\mathbb{T}$-многообразия плотны в $\mathscr{H}(\mathbb{R} ; g, 1)$.

2. Альтернанс и накрытия. В этом пункте для исследования задач наименьшего отклонения применен формализм накрытий [7]. В качестве приложения этой техники приведено чисто геометрическое доказательство теоремы 2.

Заметим, что многочлен Чебьшева $T_{n}\left(E^{+}, x\right)$ определяет $n$-листное голоморфное разветвленное накрытие сферы Римана $S$ другой сферой Римана $X$. Из теоремы 1 следует, что это накрытие разветвлено с порядком 2 во всех прообразах точек $\pm 1 \in S$, кромеграничных точек (4) компонент опорного множества $E^{+}$. Другие задачи наименьшего отклонения - в силу теоремы Чебьшева об альтернансе и ее обобщений - также приводят к накрытиям, разветвленньм над точками \pm 1 . Как видим, экстремальные многочлены являются решениями следуюшей более общей задачи [8].

ЗАДАЧА о РАЗВЕТВЛЕННЫХ НАКРЫТИЯХ. Даны две компактные римановы поверхности $X, S$; на последней отмечены точки $s_{1}, \ldots, s_{p}$. Перечислить все голоморфные отображения $\mathscr{F}: X \rightarrow S$, индексы ветвления которых в прообразах $\mathscr{F}^{-1}\left(s_{m}\right), m=1, \ldots, p$, образуют (неупорядоченные) списки $\left\{k_{m}^{i}\right\}, i=1, \ldots, l(m), \sum_{i} k_{m}^{i}=\operatorname{deg} \mathscr{F}$.

Для нахождения частных решений $\mathscr{F}$ этой задачи в [8] предлагается находить поднятия $\widetilde{\mathscr{F}}$ этих решений на специально подобранные накрьвающие поверхности $\widetilde{X}, \widetilde{S}$ диаграммы (9a).

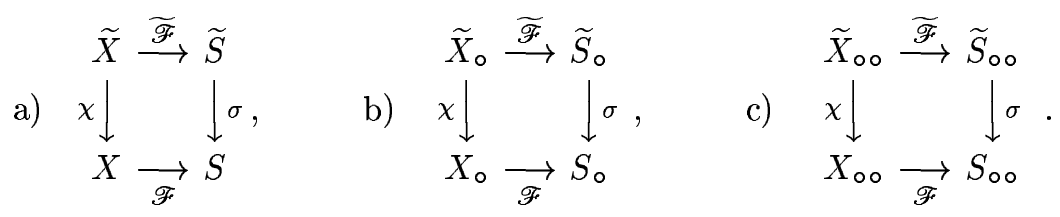

Пусть $\widetilde{S}$ разветвляется над каждой точкой $s_{m} \in S, m=1, \ldots, p$, с порядком $k_{m}$, делящимся на все элементы списка $\left\{k_{m}^{i}\right\}, i=1, \ldots, l(m)$, а $\widetilde{X}$ разветвляется над каждой точкой $x_{m}^{i} \in \mathscr{F}^{-1}\left(s_{m}\right)$ с порядком $k_{m} / k_{m}^{i}, i=1, \ldots, l(m), m=1, \ldots, p$. Если $\mathscr{F}$ можно поднять до отображения $\widetilde{\mathscr{F}}$ накрьвающих пространств (т.е. диаграмма (9а) должна быть коммутативна), то $\widetilde{\mathscr{F}}$ неразветвлено над точками $\widetilde{S}$, накрьвающими $s_{m}$, и решение $s=\mathscr{F}(x)$ представимо в параметрическом виде

$$
s=\sigma \circ \widetilde{\mathscr{F}}(\widetilde{x}), \quad x=\chi(\widetilde{x}), \quad \widetilde{x} \in \widetilde{X}
$$

В случае регулярных разветвленнных накрытий $\chi, \sigma$ с разрьвно [4], [9] действующими на накрывающих $\widetilde{X}, \widetilde{S}$ группами скольжений $\mathfrak{G}(\chi)$ и $\mathfrak{G}(\sigma)$ всякое әквивариантное отображение $\widetilde{\mathscr{F}}$ накрывающих, т.е. такое, что

$$
\widetilde{\mathscr{F}}(G \widetilde{x})=\mathbf{T}(G) \widetilde{\mathscr{F}}(\widetilde{x}), \quad \widetilde{x} \in \widetilde{X}, \quad G \in \mathfrak{G}(\chi),
$$

для некоторого гомоморфизма $\mathbf{T}: \mathfrak{G}(\chi) \mapsto \mathfrak{G}(\sigma)$, опускается до отображения факторпространств

$$
\mathscr{F}: X=\widetilde{X} / \mathfrak{G}(\chi) \rightarrow \widetilde{S} / \mathfrak{G}(\sigma)=S .
$$


При фиксированном $\widetilde{x} \in \widetilde{X}$ индекс ветвления $k_{\mathscr{F}}$ этого отображения в точке $\chi(\widetilde{x})$ можно найти из соотношения

$$
k_{\mathscr{F}} i_{\chi}=k_{\widetilde{F}} i_{\sigma}
$$

в котором $k_{\widetilde{F}}-$ индекс ветвления $\widetilde{\mathscr{F}}$ в точке $\widetilde{x} ; i_{\chi}$ и $i_{\sigma}-$ порядки стабилизаторов точек $\widetilde{x}$ и $\widetilde{s}:=\widetilde{\mathscr{F}}(\widetilde{x})$ в соответствующих групшах скольжений.

На практике накрытия $\chi, \sigma$ выбираются на основе априорной информации о решении $\mathscr{F}$ и следующей ниже леммы 1 . Пусть поверхность $S$ 。 получена выкальванием из $S$

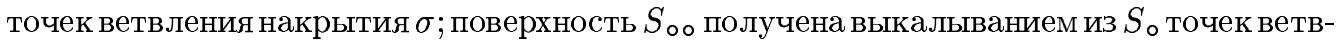
ления отображения $\mathscr{F} \circ \chi$. Положим

$$
\begin{aligned}
\widetilde{S}_{\circ}:=\sigma^{-1}\left(S_{\circ}\right), & X_{\circ}:=\mathscr{F}^{-1}\left(S_{\circ}\right), & \tilde{X}_{\circ}:=\chi^{-1}\left(X_{\circ}\right), \\
\widetilde{S}_{\circ \circ}:=\sigma^{-1}\left(S_{\circ \circ}\right), & X_{\circ \circ}:=\mathscr{F}^{-1}\left(S_{\circ \circ}\right), & \widetilde{X}_{\circ \circ}:=\chi^{-1}\left(X_{\circ \circ}\right) .
\end{aligned}
$$

Лемма 1. а) Если в диаграмме (9а) б-регулярное разветвленное голоморфное

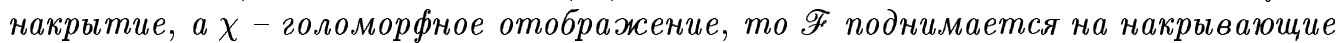
пространства тогда и только тогда, когда в группе $\pi_{1}\left(S_{\circ}\right)$ выполнено влохсение

$$
(\mathscr{F} \circ \chi) \pi_{1}\left(\tilde{X}_{\circ}\right) \subset \sigma \pi_{1}\left(\widetilde{S}_{\circ}\right)
$$

б) Если ұ - также регулярное разветвленное накрытие, то поднятое отображение $\widetilde{\mathscr{F}}$ будет әквивариантным с гомоморфизмом

$$
\mathbf{T}: \mathfrak{G}(\chi) \cong \pi_{1}\left(X_{\circ \circ}\right) / \chi \pi_{1}\left(\widetilde{X}_{\circ \circ}\right) \stackrel{\mathscr{F}}{\mapsto} \pi_{1}\left(S_{\circ \circ}\right) / \sigma \pi_{1}\left(\widetilde{S}_{\circ \circ}\right) \cong \mathfrak{G}(\sigma)
$$

Опущенные в обозначениях фундаментальных групп отмеченные точки выбраны согласованно с отображсениями диаграмм (9b), (9c).

ДокАЗАТЕЛЬСТво. а) Убедимся прежде всего, что $S \backslash S$ 。 состоит из конечного числа точек. В силу разрывности действия группы скольжений $\mathfrak{G}(\sigma)$ на накрьвающей $\widetilde{S}$ точки накрьвающей с нетривиальными стабилизаторами расположены дискретно на $\widetilde{S}$. Проецируя малые окрестности этих точек на $S$, убеждаемся, что каждая выколотая точка $S$ 。 имеет окрестность, не содержащую других выколотых точек. Из компактности $S$ следует конечность числа проколов.

Отображение $\sigma$ в диаграмме (9b) является (неразветвленным) накрытием, поэтому

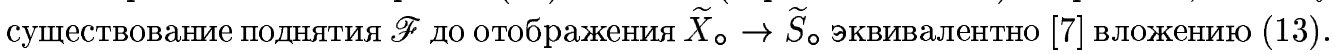

Продолжим $\widetilde{\mathscr{F}}$ в проколы поверхности $\tilde{X}_{\text {。 }}$. Последние расположены дискретно на $\widetilde{X}$, поскольку их образ при (непостоянном голоморфном) отображении $\mathscr{F} \circ \chi$ состоит из ко-

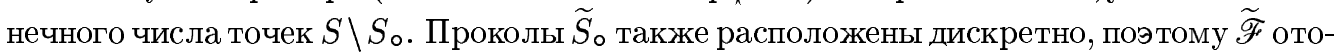
бражает достаточно малую окрестность прокола $\widetilde{x} \in \widetilde{X}$ на окрестность единственной точки $\widetilde{s} \in \widetilde{S}$ с нетривиальньм стабилизатором. Положим по непрерьвности $\widetilde{\mathscr{F}}(\widetilde{x})=\widetilde{s}$.

б) Отображения $\chi, \sigma$ диаграммы (9c) являются (неразветвленньми) регулярными накрытиями, так что фундаментальные групшы баз действуют на накрывающих пространствах и справедливы [7] представления

$$
\mathfrak{G}(\chi)=\pi_{1}\left(X_{\circ \circ}\right) / \chi \pi_{1}\left(\tilde{X}_{\circ \circ}\right), \quad \mathfrak{G}(\sigma)=\pi_{1}\left(S_{\circ \circ}\right) / \sigma \pi_{1}\left(\widetilde{S}_{\circ \circ}\right)
$$


Если отображение $\mathscr{F}$ поднимается на накрывающие $\widetilde{X}_{\text {оо }}, \widetilde{S}_{\text {оо }}$, то наряду с (13) имеет место [7] включение $(\mathscr{F} \circ \chi) \pi_{1}\left(\widetilde{X}_{\circ \circ}\right) \subset \sigma \pi_{1}\left(\widetilde{S}_{\circ \circ}\right)$, поэтому гомоморфизм Т в $(14)$ опреде-

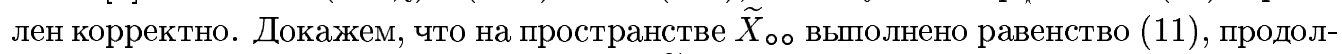
жая которое по непрерывности в проколы $\widetilde{X}_{\text {оо }}$ мы прийдем к эквивариантности поднятого отображения. Фиксируем $\widetilde{x} \in \widetilde{X}_{\text {оо }}$ путь $\tau \subset \widetilde{X}_{\text {о }}$, соединяющий отмеченную точку

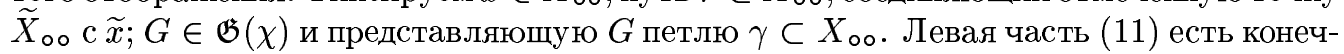

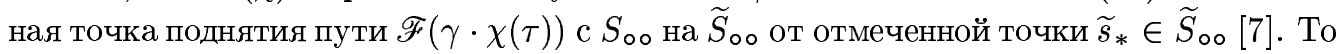
же самое значение можно получить, поднимая от $\widetilde{s}_{*}$ путь $\mathscr{F}(\gamma)$ и затем от его конечной точки - путь $\mathscr{F} \circ \chi(\tau)$. Именно так действует $\mathbf{T}(G)$ на точку $\widetilde{\mathscr{F}}(\widetilde{x})$.

ДокаЗАТЕЛЬСТво ТЕоремы 2. 1) Фиксируем многочлен Чебьшева $T_{n}\left(E^{+}, x\right)$. Отображение $\mathscr{F}: X:=\mathbb{C P}_{1} \stackrel{T_{n}}{\rightarrow} \mathbb{C P}_{1}=: S$ неразветвлено в накрьваюших $\pm 1 \in S$ точках $-1, a_{1}, b_{1}, \ldots, a_{g}, b_{g}, 1 \in X$ и разветвлено со вторым порядком во всех остальных точках множества $T_{n}^{-1}( \pm 1)$. Регулярное накрытие $\widetilde{X}:=M\left(E^{+}\right) \stackrel{\chi}{\rightarrow} X-$ это в известном смысле "минимальное" накрытие, ветвящееся с порядком 2 над указанными вьше $2 g+2$ точками $X$; естественная проекция $\chi: \widetilde{x}=(x, w) \rightarrow x$ имеет группу скольжений, порожденную инволюцией $J:(x, w) \rightarrow(x,-w)$. В качестве ветвящейся над $\pm 1 \in S$ накрывающей возьмем $\widetilde{S}:=\mathbb{C P}_{1} ;$ накрытие $\sigma: \widetilde{s} \rightarrow(\widetilde{s}+1 / \widetilde{s}) / 2$ имеет группу скольжений, порожденную отображением $\widetilde{s} \rightarrow 1 / \widetilde{s}, \widetilde{s} \in \widetilde{S}$.

Покажем, что отображение $\mathscr{F}$ можно поднять на накрываюшие пространства, для чего проверим вьполнение вложения (13). Фундаментальная группа $\pi_{1}\left(S_{\circ}\right)$ - это свободная группа с одной образующей. Ее подгрупша $\sigma \pi_{1}\left(\widetilde{S}_{\circ}\right)$ порождена квадратом этой образующей. Выберем в $\pi_{1}\left(\widetilde{X}_{\circ}\right)$ базис, элементы которого представлены петлями двух видов: $4 n$ петель первого вида - лассо, зацепленных за проколы в римановой поверхности $\widetilde{X}_{\text {о }}$, и $2 g$ петель второго вида, гомотопных циклам канонического базиса $\mathbf{A}_{1}, \ldots, \mathbf{A}_{g}$; $\mathbf{B}_{1}, \ldots, \mathbf{B}_{g}$. Индекс ветвления отображения $\mathscr{F} \circ \chi$ равен 2 в каждом из проколов $\tilde{X}_{\circ}$, поэтому петли первого вида проецируются в лассо, дважды огибающие один из про-

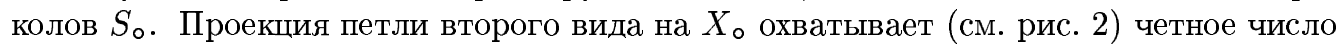
точек (4), а также некоторое число других прообразов $T_{n}^{-1}( \pm 1)$. Продеформируем эту

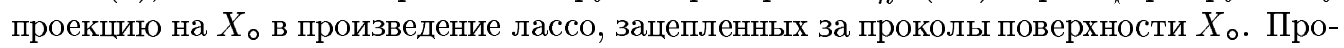

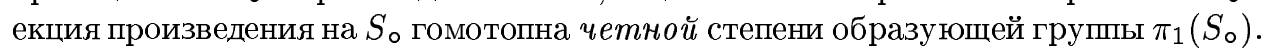

Поднятие $\mathscr{F}$ на накрьвающие пространства определено с точностью до движений из $\mathfrak{G}(\sigma)$, а эквивариантность $\widetilde{\mathscr{F}}$ означает в нашем случае

$$
\widetilde{\mathscr{F}}(J t)=\frac{1}{\widetilde{\mathscr{F}}(t)}, \quad t \in \widetilde{X} .
$$

Определим на накрывающей $\widetilde{X}:=M\left(E^{+}\right)$абелев дифференциал третьго рода:

$$
d \eta=\frac{d \widetilde{\mathscr{F}}}{n \widetilde{\mathscr{F}}}
$$

полюсы которого ввиду коммутативности (9a) совпадают с полюсами функции $\mathscr{F} о \chi$, т.е. находятся в бесконечно удаленных точках верхнего $\infty_{+}$и нижнего $\infty_{-}$листов $M\left(E^{+}\right)$. Устраняя двузначность в выборе $\widetilde{\mathscr{F}}$, считаем

$$
\text { Res }\left.d \eta(t)\right|_{t=\infty_{ \pm}}=\mp 1 .
$$


Остается определить периоды $d \eta$. Деформируя контур $\mathbf{A}_{s}$ в отрезок $\left[a_{s}, b_{s}\right]$, проходимый сначала по нижнему листу $M$ и обратно по верхнему листу, убеждаемся, что

$$
\int_{\mathbf{A}_{s}} d \eta=\left.\frac{1}{n} \ln \widetilde{\mathscr{F}}\right|_{\mathbf{A}_{s}}=\left.\frac{i}{n} \arg \widetilde{\mathscr{F}}\right|_{\mathbf{A}_{s}}=0, \quad s=1, \ldots, g .
$$

Аналогично, цикл $\widetilde{\mathbf{B}}_{k}$ продеформируем в отрезок $\left[b_{k}, a_{k+1}\right]$, проходимьй сначала по нижнему берегу и обратно по верхнему. Когда $\widetilde{x} \in \widetilde{X}$ пробегает по указанному контуру, точка $\widetilde{s}=\widetilde{F}(\widetilde{x})$ ровно $m_{k}$ раз описьвает окружность $|\widetilde{s}|=1$ против часовой стрелки, следовательно,

$$
\int_{\widetilde{\mathbf{B}}_{k}} d \eta=\left.\frac{i}{n} \arg \widetilde{\mathscr{F}}\right|_{\widetilde{\mathbf{B}}_{k}}=\frac{2 \pi i m_{k}}{n}, \quad k=0,1, \ldots, g .
$$

Представление (7) следует из определения (16) и коммутативности (9a).

2) Пусть, обратно, риманова поверхность $M\left(E^{+}\right)(5)$ такова, что вьполнены соотношения (6) для $\mathbf{A}$ - нормированного дифференциала третьего рода

$$
d \eta=\frac{d x}{w} \prod_{k=1}^{g}\left(x-c_{k}\right), \quad c_{k} \in\left(a_{k}, b_{k}\right) .
$$

На $M\left(E^{+}\right)$корректно определена ввиду (6) функция

$$
\widetilde{\mathscr{F}}(t)=\exp n \int_{t_{0}}^{t} d \eta, \quad t \in M, \quad t_{0}=(1,0) \in M,
$$

удовлетворяющая условию эквивариантности (15), поскольку $d \eta$ меняет знак при инволюции $J$, тождественно действующей на $t_{0}$. Порожденное $\widetilde{\mathscr{F}}$ отображение факторпространств

$$
\mathscr{F}: X=\widetilde{X} / \mathfrak{G}(\chi) \stackrel{\widetilde{F}}{\rightarrow} \widetilde{S} / \mathfrak{G}(\sigma)=S
$$

параметрически записывается в виде $(10)$ :

$$
\mathscr{F}(x)=\sigma \circ \widetilde{\mathscr{F}}(t)=\cos n i \int_{t_{0}}^{t} d \eta, \quad x=\chi(t) \in X, \quad t \in \tilde{X},
$$

и является рациональной функцией с единственным полюсом степени $n$ на бесконечности, т.е. полиномом.

Остается показать, что интервал $\left(b_{k}, a_{k+1}\right)$ составлен из $m_{k}$ интервалов, каждый из которых $\mathscr{F}(x)$ взаимно однозначно отображает на $I$. Действительно, при уменьшении $x$ от $a_{k+1}$ до $b_{k}$ аргумент косинуса в представлении (7) монотонно, что следует из (18), изменяется на $\pi m_{k}$. На краях $b_{k}, a_{k+1}$ аргумент кратен $\pi$ - это выводится индукцией по $k$ из $\mathbf{A}$-нормированности дифференциала $d \eta$. Поскольку числа $m_{k}$ образуют разбиение степени $n$ многочлена $\mathscr{F}(x)$, то из теоремы 1 следует, что $T_{n}:=\mathscr{F}(x)-$ многочлен Чебышева с опорным множеством $E^{+}$.

3. Геометрия многообразия опорных множеств. Сформулируем лемму, следствием которой является теорема 3 . Пусть $d \eta$ - абелев дифференциал третьего рода (18) на римановой поверхности (5), не обязательно нормированньй. Определим отображение $\left\{a_{k}, b_{k}, c_{k}\right\}_{k=1}^{g} \rightarrow\left\{\lambda_{k}, \mu_{k}, \nu_{k}\right\}_{k=1}^{g}$ при помощи равенств

$$
\lambda_{k}:=\int_{\mathbf{A}_{k}} d \eta, \quad \mu_{k}:=-i \int_{\mathbf{B}_{k}} d \eta, \nu_{k}:=\int_{\mathbf{C}_{k}} d \eta, \quad k=1, \ldots, g,
$$

пути $\mathbf{A}_{k}, \mathbf{C}_{k}$ указаны на рис. $2, \mathbf{B}_{k}:=\widetilde{\mathbf{B}}_{0}+\widetilde{\mathbf{B}}_{1}+\cdots+\widetilde{\mathbf{B}}_{k-1}$. 
Лемма 2. Отображение (19) является вещественно-аналитическим диффеоморфизмом $3 g$-мерного симплекса

$$
-1<a_{1}<c_{1}<b_{1}<a_{2}<\cdots<c_{g}<b_{g}<1
$$

на произведение g-мерного симплекса и $2 g$-мерного конуса

$$
0<\mu_{1}<\mu_{2}<\cdots<\mu_{g}<2 \pi, \quad \lambda_{k}<\nu_{k}, \quad 0<\nu_{k}, \quad k=1, \ldots, g .
$$

ДокАЗАТЕЛЬСТво. Интеграл Кристоффеля-Шварца

$$
2 i \int_{-1}^{x} d \eta, \quad \operatorname{Im} x>0
$$

конформно отображает верхнюю полуплоскость на гребенчато-ступенчатую область, показанную на рис. 3. Ее параметры взаимно однозначно связаны с величинами $\left\{\lambda_{k}, \mu_{k}\right.$, $\left.\nu_{k}\right\}$, например: абсцисса $k$-го вертикального разреза есть $\mu_{k}$; высота $k$-го разреза относительно следующей за ним горизонтальной площадки есть $\nu_{k}$, разность уровней горизонтальных плошадок, примыкаюших к $k$-му разрезу, есть $\lambda_{k}$. Обратное отображение области (21) на симплекс (20) определим при помощи подходящим образом нормированного конформного отображения гребенчато-ступенчатых областей рис. 3 на верхнюю полуплоскость. Покажем, что биективное отображение $\left\{a_{k}, b_{k}, c_{k}\right\} \longleftrightarrow\left\{\lambda_{k}, \mu_{k}, \nu_{k}\right\}$ является в обе стороны вещественно-аналитическим.

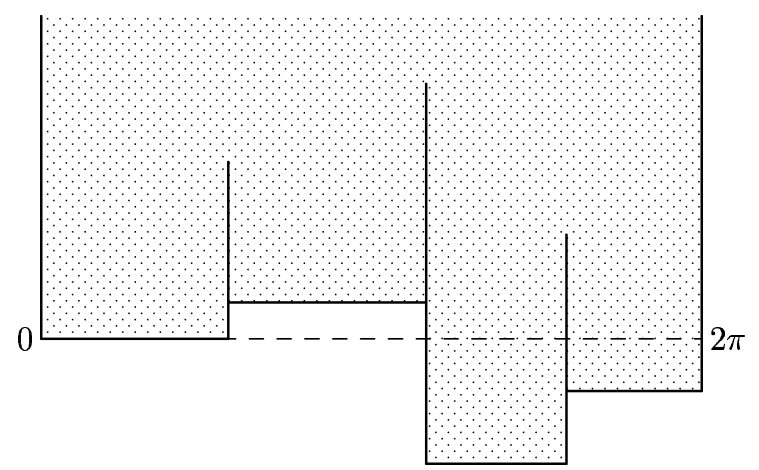

Рис. 3. Гребенчато-ступенчатая область при $g=3$

Дифференцируя выражения для $\lambda, \mu, \nu$ по параметрам $a, b, c$, получим следуюшее выражение для матрицы Якоби отображения (19):

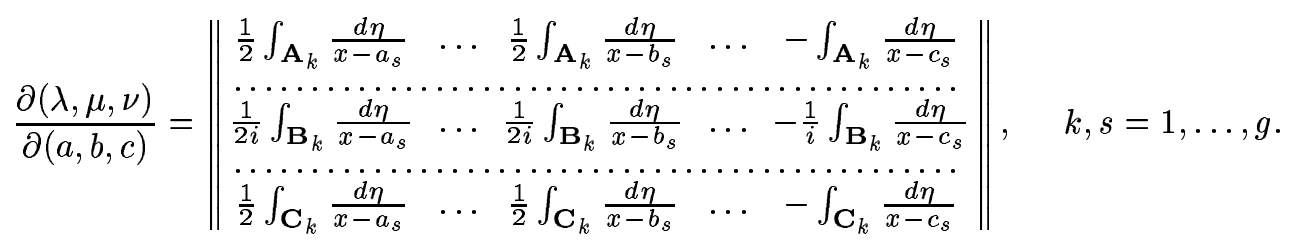

Вырожденность матрицы Якоби означает, что найдется ненулевой дифференциал второго рода вида

$$
d \omega:=\sum_{s=1}^{g}\left(\frac{\alpha_{s}}{x-a_{s}}+\frac{\beta_{s}}{x-b_{s}}+\frac{\gamma_{s}}{x-c_{s}}\right) d \eta
$$


с постоянными $\alpha_{s}, \beta_{s}, \gamma_{s}$, для которого интегралы по всем кривьм $\mathbf{A}_{k}, \mathbf{B}_{k}, \mathbf{C}_{k}$ равнынулю. Заметим, что полюсы $d \omega$ могут располагаться только в точках $\left(a_{s}, 0\right),\left(b_{s}, 0\right) \in M$, причем вычеты $d \omega$ в полюсах равны нулю: $d \omega$ меняет знак при инволюции $J$, оставляющей неподвижными точки ветвления $M$. Абелев интеграл

$$
\omega(t)=\int_{t_{0}}^{t} d \omega, \quad t_{0}=(1,0) \in M
$$

однозначен на $M$, поскольку и циклические и полярные периоды $d \omega$ равны нулю. Функция $\omega$ меняет знак при инволюции $J$ ввиду нечетности дифференциала $d \omega$, поэтому $\omega=0$ во всех точках $t_{k} \in M$, накрьвающих точки $c_{k}, k=1, \ldots, g$. Функция $w \omega \in \mathbb{C}(M)$ инвариантна при инволюции $J$ и имеет два полюса на бесконечности порядка не вьше $g+1$ в качестве единственных особенностей, поэтому

$$
w(t) \omega(t)=P_{g+1}(x), \quad x=\chi(t), \quad t \in M,
$$

где полином $P_{g+1}$ имеет $g+2$ корня $\pm 1, c_{1}, \ldots, c_{g}$. Следовательно, $\omega \equiv 0$ и матрища Якоби невырождена. Теорема об обратной функции теперь гарантирует аналитичность отображения $\{\lambda, \mu, \nu\} \rightarrow\{a, b, c\}$.

ДОКАЗАТЕЛЬСТВО ТЕОРЕМЫ 3. Пространство модулей $\mathscr{H}(\mathbb{R} ; g, 1)$ естественно вкладывается в $3 g$-мерньй симплекс (20) в качестве решений системы уравнений $\lambda_{1}=\lambda_{2}=$ $\cdots=\lambda_{g}=0$, при этом система чисел (4) дополняется нулями $c_{k} \quad \mathbf{A}$-нормированного дифференциала третьего рода $d \eta$ с полюсами на бесконечности. Подмногообразие $\mathbb{T}\left(m_{0}\right.$, $\left.\ldots, m_{g}\right) \subset \mathscr{H}(\mathbb{R} ; g, 1)$ - это множество, выделяемое $g$ дополнительными равенствами $\mu_{k}=2 \pi\left(m_{0}+m_{1}+\cdots+m_{k-1}\right) / n, k=1, \ldots, g$, с $n$ из (2). Отсюда легко вытекают все утверждения теоремы.

\section{СПИСОК ЦИТИРОВАННОЙ ЛИТЕРАТУРЫ}

[1] Peherstorfer F. Minimal polynomials on several intervals with respect to the maximum-norm -a survey // Proc. of the Workshop "Methods of Complex Analysis in Approximation Theory": Almeria, 1995. P. 137-160.

[2] Содин М. Л., Юдицкий П. М. Функции, наименее уклоняющиеся от нуля на замкнутых подмножествах действительной оси // Алгебра и анализ. 1992. Т. 4. № 2. С. 1-61.

[3] Peherstorfer F. On Bernstein-Szegö orthogonal polynomials on several intervals. II // J. Appr. Theory. 1991. V. 64. P. 123-161.

[4] Farkas H. M., Kra I. Riemann Surfaces. 2nd edition: Springer GTM, 71.

[5] Ахиезер Н. И. Über einige Funktionen die in gegeben Intervallen am wenigsten von Null abweichen // Изв. Казанского физ. -матем. общества. 1928. Т. 3. № 3. С. 1-69.

[6] Ахиезер Н.И., Левин Б.Я. Неравенства для производных, аналогичные неравенству Бернштейна // Докл. АН СССР. 1957. Т. 117. № 5. С. 735-738.

[7] Фоменко А.Т., Фукс Д. Б. Курс гомотопической топологии. М.: Наука, 1989.

[8] Богатырев А.Б. Интегральные уравнения Пуанкаре-Стеклова и задачи монодромии // Функцион. анализ и прилож. 2000. Т. 34. № 2.

[9] Апанасов Б. Н. Геометрия дискретных групп и многообразий. М.: Наука, 1991.

Институт вычислительной математики РАН

Поступило Московский физико-технический институт 